\title{
Hydrazine-Modified Starch Coated Magnetic Nanoparticles as an Effective pH-Responsive Nanocarrier for Doxorubicin Delivery
}

\author{
Nasrin Zohreh, ${ }^{1 *}$ Seyed Hassan Hosseini ${ }^{2}$, and Ali Pourjavadi ${ }^{2}$ \\ ${ }^{1}$ Department of chemistry, Faculty of Science, University of Qom, P. O. Box: 37185-359, Qom, Iran \\ ${ }^{2}$ Polymer Research Laboratory, Department of Chemistry, Sharif University of Technology, Tehran, Iran \\ (Corresponding Author: Dr. Nasrin Zohreh, n.zohreh@qom.ac.ir; Phonelfax: +982532103488)
}




\begin{abstract}
A novel, magnetic nanocarrier was successfully synthesized through a facile and economical producer in which $\mathrm{Fe}_{3} \mathrm{O}_{4}$ magnetic nanoparticles were coated by starch-g-poly (methyl methacrylate-co-PEG-acrylamide). The surface of nanocarrier was then modified by hydrazine to preparation of a $\mathrm{pH}$-responsive carrier. The resulted nanocarrier was applied for delivery of doxorubicin (DOX) as an effective anti-cancer drug. DOX was reacted with hydrazine linkage on the surface of nanocarrier to form hydrazone bond. Due to the presence of numerous hydrazine groups on the surface of magnetic nanocarrier large amounts of DOX was loaded onto the carrier $\left(327 \mathrm{mg} \mathrm{g}^{-1}\right)$.
\end{abstract}

\title{
KEYWORDS
}

Magnetic nanocarrier, Drug delivery, Doxorubicin, Starch, $\mathrm{pH}-$ Senstive 


\section{INTRODUCTION}

Over the last decade, magnetic nanoparticles (MNPs) received many attentions in cancer therapy due to they can be targeted to tumor cells using an external magnet. ${ }^{1-11}$ They also can be used as contrast agents for magnetic resonance imaging (MRI) because they impel a shorter $T_{2}$ relaxation, producing decreased signal intensity on a $T_{2}$-weighted image. ${ }^{2,12}$ The imaging enhancement property conjugated with magnetic targeted drug delivery makes MNPs very interesting as anti-cancer nanocarrier for real-time monitoring of drug distribution in cancer cells, as well as to investigate the effect of loaded drags on the size of tumor. ${ }^{7,13-18}$ Delivery of anti-cancer drugs by magnetic carriers solves the problem of insolubility of hydrophobic anticancer drugs in blood medium and reduces the serious side effects caused by cytotoxicity of anticancer drugs towards normal cells.

There are several reports that MNPs have been coated with polymers and loaded with therapeutic agents for targeted drug delivery. ${ }^{1-8,19-21}$ In these magnetic carriers anti-cancer drugs were either covalently bonded to specific functional groups on the surface of MNPs or physically interacted to the surface of modified MNPs by electrostatic or hydrophobic interactions. Coating of the surface of MNPs with biocompatible polymers such as polysaccharides and PEG reduces reticuloendothelial system (RES) uptake as well as nonspecific interaction with plasma membranes. ${ }^{22-28}$ However, these simple coatings with polysaccharides and PEG cannot induce the appropriate sensitivity for selective release of anti-cancer drugs to cancer cells and tissues. Since, cancer cells have lower $\mathrm{pH}$ and higher temperature than normal cells, using stimuli responsive polymers as coating for MNPs can selectively increase the drug concentrations in cancer cells and extremely decrease the side effects. ${ }^{29-35}$ Another important aspect for in vivo drug delivery is stability of nanocarrier suspensions at physiological conditions and elimination 
of nanocarrier after long time blood circulation. Many studies showed that the uptake of PEGylated MNPs by immune system is lower than for non-PEGylated ones. ${ }^{36-38}$ This resulted to longer blood circulating lifetimes of magnetic nanocarriers, which favors their accumulation in tumor sites by both magnetically guiding and the enhanced permeability and retention (EPR) effect.

DOX is a highly efficient antineoplastic agent commonly used in the treatment various types of cancers. ${ }^{39,40}$ The clinical use of DOX is limited by the resistance developed by cancer cells and by strong toxicity to normal cells which caused to deadly side effects. ${ }^{41,42}$ Targeted delivery of DOX to cancer tissues is a rationalized way to decrease the concentration of DOX in normal cells while the concentration of drug in cancer cells is still high.

In the present work, we prepared a multifunctional $\mathrm{pH}$-sensitive magnetic carrier for in vitro DOX delivery. First, starch as a biocompatible polysaccharide was modified by PEG and hydrazine groups and then this modified starch was coated onto the $\mathrm{Fe}_{3} \mathrm{O}_{4}$ magnetic nanoparticles. Afterward, DOX molecules were conjugated to this magnetic carrier by hydrazone bond which this linkage is $\mathrm{pH}$-sensitive and can be ruptured in acidic medium. The release of DOX and cytotoxic effect of magnetic carrier were investigated on HeLa cells. The results showed that the present magnetic carrier can be a suitable choice for in vivo delivery of DOX.

\section{MATERIALS AND METHODS}

\section{Materials and Instruments}

Ferric chloride hexahydrate $\left(\mathrm{FeCl}_{3} \cdot 6 \mathrm{H}_{2} \mathrm{O}\right)$, ferrous chloridetetrahydrate $\left(\mathrm{FeCl}_{2} \cdot 4 \mathrm{H}_{2} \mathrm{O}\right)$, Ammonium hydroxide (30\%) and 3-aminopropyltriethoxysilane (APTS) obtained from Merck. Methyl acrylate monomer (Aldrich) was distilled using hydroquinone as polymerization 
inhibitor. Hydrazine monohydrate (100\%), starch and methyl methacrylate (MMA) were purchased from Aldrich. Methoxypolyethylene glycol amine $\left(M_{w}=1000 \mathrm{~g} / \mathrm{mol}\right)$ was received from Alfa Aesar. Doxorubicin hydrochloride was obtained from Pfizer, China. All other materials and solvents were obtained from Merck and double distilled water was used for experiments. Dialysis bag (molecular weight cutoff: $3.5 \mathrm{KDa}$; diameter: $22 \mathrm{~mm}$ ) was purchased from Beijing Solarbio Science \& Technology Co., Ltd., China. HeLa cells were donated by Iran Pasteur Institute, Tehran, Iran. RPMI medium and fetal bovine serum (FBS) were obtained from Biochrom AG, Germany. Dimethyl sulfoxide (DMSO) and [3-(4,5-dimethylthiazol-2-yl)-2,5diphenyltetrazolium bromide] (MTT) were purchased from Sigma. Deionized water was used in all the experiments.

UV-Vis spectra were collected using a Perkin-Elmer spectrophotometer. FT-IR spectra were recorded on ABB Bommem MB-100 spectrometer (Canada), KBr was used for making pallet of samples. Thermogravimetric analysis (TGA) was acquired under a nitrogen atmosphere with a heating rate $10{ }^{\circ} \mathrm{C} . \mathrm{min}^{-1}$ using a TGA Q50 thermo-gravimetric analyzer . The XRD pattern was recorded on a RigalcuD/Max-3c X-ray diffractometer. The sizes of samples were observed using transmission electron microscopy (TEM) taken with a Philips CM30 electron microscope. Dynamic light scattering (DLS) measurements were performed by using Zetaplus/90plus instrument (Brookhaven Instrument Co. USA). Magnetizations of samples were measured by vibrating sample magnetometer (Meghnatis Daghigh Kavir Co., Kashan, Iran). Molecular weight of polymers were determined by Agilent 1100 gel permeation chromatograph (GPC) equipped with a refractive index detector using DI water as the eluent at a flow rate of $1 \mathrm{~mL} \mathrm{~min}^{-1}$ at 30 ${ }^{\circ} \mathrm{C}$. Dextran was used as standard sample for molecular weight measurement in GPC. 


\section{Synthesis of macromonomer PEG-methacrylamide mono methyl ether}

In a $50 \mathrm{~mL}$ round bottom flask, $3.4 \mathrm{~g}$ methoxypolyethylene glycol amine was dissolved in $30 \mathrm{~mL}$ methanol. Then, an excess amount of methyl methacrylate $(1.2 \mathrm{~g})$ was added to solution and mixture was refluxed for $30 \mathrm{~h}$ under vigorous stirring. After completion of the reaction, the mixture was concentrated by evaporation of solvent. Then, product (PEG-Am) was precipitated in $100 \mathrm{~mL}$ diethyl ether to remove excess amount of methyl methacrylate.

\section{Synthesis of starch-g-poly(methyl methacrylate-co-PEG-Am)}

The synthesis of grafted copolymer was performed using persulfate/ascorbic acid redox pair at low temperature to prevent the hydrolysis of methyl ester groups in high temperature polymerization. ${ }^{43}$ Typically, in a $100 \mathrm{~mL}$ three-necked round bottom flask equipped with a condenser, $1.0 \mathrm{~g}$ starch, $0.03 \mathrm{~g}$ ascorbic acid as redox initiator were dissolved in $50 \mathrm{~mL}$ water at $35^{\circ} \mathrm{C}$. Then, $0.2 \mathrm{~g} \mathrm{~K}_{2} \mathrm{~S}_{2} \mathrm{O}_{8}$ was added to solution and the temperature of the solution adjusted to $35^{\circ} \mathrm{C}$. After $10 \mathrm{~min}$, a mixture of $0.5 \mathrm{~g}$ methyl methacrylate and $0.3 \mathrm{~g}$ PEG-Am in $10 \mathrm{~mL}$ water were drop-wise added to solution. The reaction continued for $2 \mathrm{~h}$ at $35{ }^{\circ} \mathrm{C}$. Grafted product was separated from the solution by pouring them to $50 \mathrm{~mL}$ ethanol. The grafted starch finally extracted with ethanol in a soxhlet apparatus for $3 \mathrm{~h}$ to remove the adhered homopolymer if any. The white solid starch-grafted- poly(MMA-co-PEG-Am) (noted as SPMP) was dried under vacuum at $30{ }^{\circ} \mathrm{C}$ for $10 \mathrm{~h}$.

\section{Preparation of magnetic carrier}

First, $\mathrm{Fe}_{3} \mathrm{O}_{4}$ magnetic nanoparticle was synthesized according to our previously reported method. ${ }^{44}$ Then, $\mathrm{Fe}_{3} \mathrm{O}_{4}$ nanoparticles were functionalized by amine groups using APTS based on 
our previous paper $\left(\mathrm{MNP} @ \mathrm{NH}_{2}\right){ }^{20}$ For coating of MNPs with SPMP, first, $0.75 \mathrm{~g}$ of SPMP polymer was dissolved in $300 \mathrm{~mL}$ water at $35{ }^{\circ} \mathrm{C}$. Then, $0.50 \mathrm{~g}$ of aqueous dispersion of $\mathrm{MNP} @ \mathrm{NH}_{2}$ was drop-wise added to polymer solution and mixture was sonicated for $30 \mathrm{~min}$. Afterward, mixture was stirred for $3 \mathrm{~h}$ at $50{ }^{\circ} \mathrm{C}$ to covalent attaching of polymer to the surface of MNPs by amide bonds. Polymer coated MNPs (MNP@SPMP) were magnetically separated and washed four times with $50 \mathrm{~mL}$ water. The MNP@SPMP nanoparticles were then subjected to reaction with hydrazine. For this reaction, the recovered MNP@SPMP (0.5 g) was ultrasonically dispersed in $100 \mathrm{~mL}$ methanol and then $4 \mathrm{~mL}$ hydrazine was added to solution and the solution was stirred at $50{ }^{\circ} \mathrm{C}$ for $18 \mathrm{~h}$. The resulted magnetic nanoparticle coated starch- $g$ poly(hydrazine methacrylate-co-PEG-Am) (noted as MNP@SPHP) was magnetically separated and washed several time with water $(5 \times 50 \mathrm{~mL})$ and methanol $(2 \times 20 \mathrm{~mL})$ and dried at $50{ }^{\circ} \mathrm{C}$.

\section{Loading of DOX onto the MNP@SPHP}

In a $25 \mathrm{~mL}$ round bottom flask, $30 \mathrm{mg}$ MNP@SPHP magnetic nanocarrier was ultrasonically dispersed into the $10 \mathrm{~mL}$ PBS buffer and then $2 \mathrm{~mL}$ DOX $\left(5 \mathrm{mg} \mathrm{mL}^{-1}\right)$ was added to solution. The flask was covered by foil (to keep solution in dark) and the mixture was stirred for $24 \mathrm{~h}$ at room temperature. Afterward, DOX loaded magnetic carrier MNP@SPHP@DOX was magnetically separated and washed with PBS buffer $(3 \times 5 \mathrm{~mL})$ until supernatant solutions became colorless. Then the supernatant solutions were collected and kept for UV-Vis analysis.

The drug loading capacity (DLC) and efficiency (DLE\%) were calculated using the following equation: 


$$
\begin{gathered}
\operatorname{DLC}=\frac{M_{I D}-\sum M_{R D}}{M_{N C}} \\
\operatorname{DLE}(\%)=\frac{M_{I D}-M_{R D}}{M_{I D}} \times 100
\end{gathered}
$$

Where $M_{\mathrm{ID}}$ is the initial DOX mass, $M_{\mathrm{RD}}$ is the residual DOX mass in the supernatant solutions and $M_{\mathrm{NC}}$ is the nanocarrier mass.

The release studies were performed at $37{ }^{\circ} \mathrm{C}$ at $\mathrm{pH}=5.0$ and 7.4 using acetate and phosphate buffer solution, respectively. Firstly, 10 mg MNP@SPHP@DOX was dispersed in 3 mL buffer solution and placed in a dialysis bag with a molecular weight cut-off of $3.5 \mathrm{kDa}$. The dialysis bag was immersed in $25 \mathrm{~mL}$ of the same buffer medium and stirred at a constant temperature. Samples $(2 \mathrm{~mL})$ were periodically sucked up and filled the same volume of fresh buffer. The amount of released DOX was analyzed with UV-Vis spectroscopy at $490 \mathrm{~nm}$. The drug release studies were performed in triplicate for each of the samples.

\section{Cytotoxicity analysis of MNP@SPHP@DOX}

The in vitro cytotoxicity assay was performed on HeLa cell and a MTT assay was used to determine the cell viability of samples. Cells were cultured in RPMI containing fetal bovine serum (FBS) $10 \%$, penicillin $100 \mathrm{mg} \mathrm{mL}^{-1}$ and streptomycin $100 \mathrm{mg} \mathrm{mL}^{-1}$ at $37{ }^{\circ} \mathrm{C}$ in a $5 \% \mathrm{CO}_{2}$ incubator for $24 \mathrm{~h}$ incubation. Then, cells were seeded onto the 48 -well plates with a concentration of 4000-5000 cells per well. The drug nanocarriers were sterilized by ultraviolet irradiation for $1 \mathrm{~h}$ and added to culture wells at different concentrations $\left(1,5,10,25,50 \mu \mathrm{g} \mathrm{mL}^{-1}\right)$ while the culture wells without nanocarriers act as control groups. Then, the solutions were 
incubated for $24 \mathrm{~h}$ at $37{ }^{\circ} \mathrm{C}$ with $5 \% \mathrm{CO}_{2}$. Afterward, $20 \mu \mathrm{L}$ of MTT solution $\left(5 \mathrm{mg} \mathrm{mL}^{-1}\right.$ in PBS) was added to each well and the plate was incubated for another $4 \mathrm{~h}$ at $37^{\circ} \mathrm{C}$. The plate was covered by aluminum foil to protect from light. In this stage, viable cells reduce the MTT to formazan which is soluble in DMSO. Afterward, the medium containing unreacted dye was removed carefully. The obtained purple formazan crystals were dissolved in $200 \mu \mathrm{L}$ per well DMSO and the absorbance was measured at a wavelength of $490 \mathrm{~nm}$. All experiments for each sample were performed in triplicate.

The following formula was used to calculate the inhibition of cell growth:

$$
\text { Cell viability }(\%)=\frac{\text { mean of abs. value of treatment sample }}{\text { mean of abs. value of control sample }} \times 100
$$

\section{RESULTS AND DISCUSSIONS}

To obtain a biocompatible magnetic nanocarrier with rapid response to acidity of cancer cells for DOX delivery we first synthesized a multifunctional biocompatible polymer as MNPs shell. Starch as a cheap, and available biocompatible polymer was chosen as a backbone for modification. It is known that starch-grafted polymers are biocompatible; therefore coating of MNPs with such biocompatible polymers reduces reticuloendothelial system uptake as well as nonspecific interaction with plasma membranes. ${ }^{45-47}$ Moreover, coating of MNPs with starchgrafted polymer improves the solubility of nanocarrier in water medium due to the presence of numerous hydroxyl groups in starch structure, so nanocarrier can longer be stable in blood circulation. Another advantage of starch as polymer backbone is increasing in drug loading capacity. Starch can be grafted in multiple points by functional polymer chains which can hold drug molecules. Modification of starch was performed through a grafting-from polymerization of 
MMA and PEG-Am monomers to produce SPMP (Scheme 1). The decomposition of persulfate produces sulfate radical which this radical directly reacts with starch and generates radicals on starch backbone. ${ }^{48}$ After drop-wise addition of monomers radical polymerization initiate from the starch backbone.

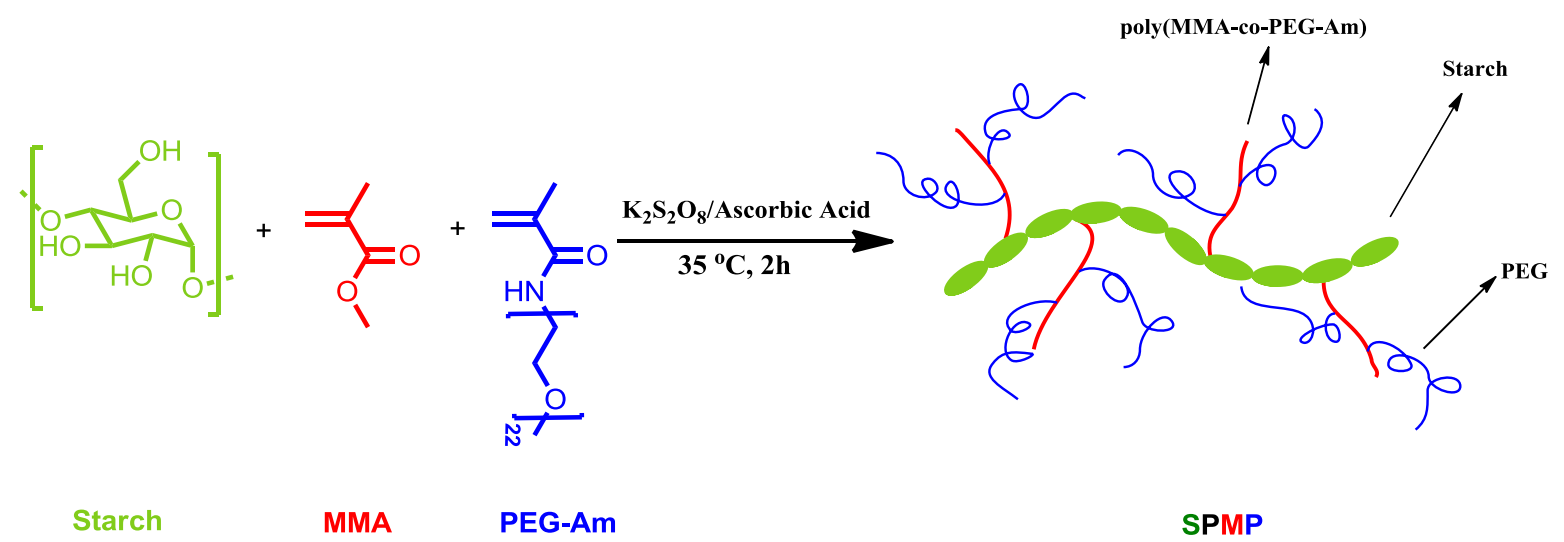

SCHEME 1 Synthesis of starch-g-poly(methyl methacrylate-co-PEG-Am)

A copolymer of poly(methyl methacrylate) was chosen for grafting to starch due to $\mathrm{CO}_{2} \mathrm{Me}$ groups in SPMP can be easily reacted to many compounds and converted to various useful functionalities for immobilization of drugs, enzymes, proteins and labeling reagents. It is known that, the $\mathrm{pH}$ of physiological media for normal cells is near 7.4 while in cancer cells it drops to around 5.5. ${ }^{49}$ To use the effect of differential $\mathrm{pH}$ values for drug release, we decided to convert $\mathrm{CO}_{2} \mathrm{Me}$ groups of grafted polymer to hydrazone linker for DOX immobilization, which conjugation of DOX-hydazone bond can be easily formed at $\mathrm{pH}$ 7.4, but hydrolyzed rapidly under acidic condition. ${ }^{27,50}$ Moreover, the presence of PEG-Am monomer in the structure of SPMP improves the biocompatibility and dispersion of final nanocarrier.

In the second step, SPMP polymer was coated around the magnetic nanoparticles (MNP@SPMP). SPMP polymer was covalently attached to the surface of MNP@ $\mathrm{NH}_{2}$ through 
the amidation reaction between amine groups of MNPs and some of methyl ester groups of SPMP polymer. Covalently attachment of polymer to the surface of MNP stabilized magnetic nanoparticles from aggregation in biological medium. Subsequently, ester groups on MNP@SPMP were reacted with hydrazine to produce desired magnetic nanocarrier (MNP@SPHP). After that, DOX was loaded onto the MNP@SPHP by strong and stable hyrazone linkage (MNP@SPHP@DOX). Scheme 2 shows the step-wise preparation of drug loaded magnetic nanocarrier.

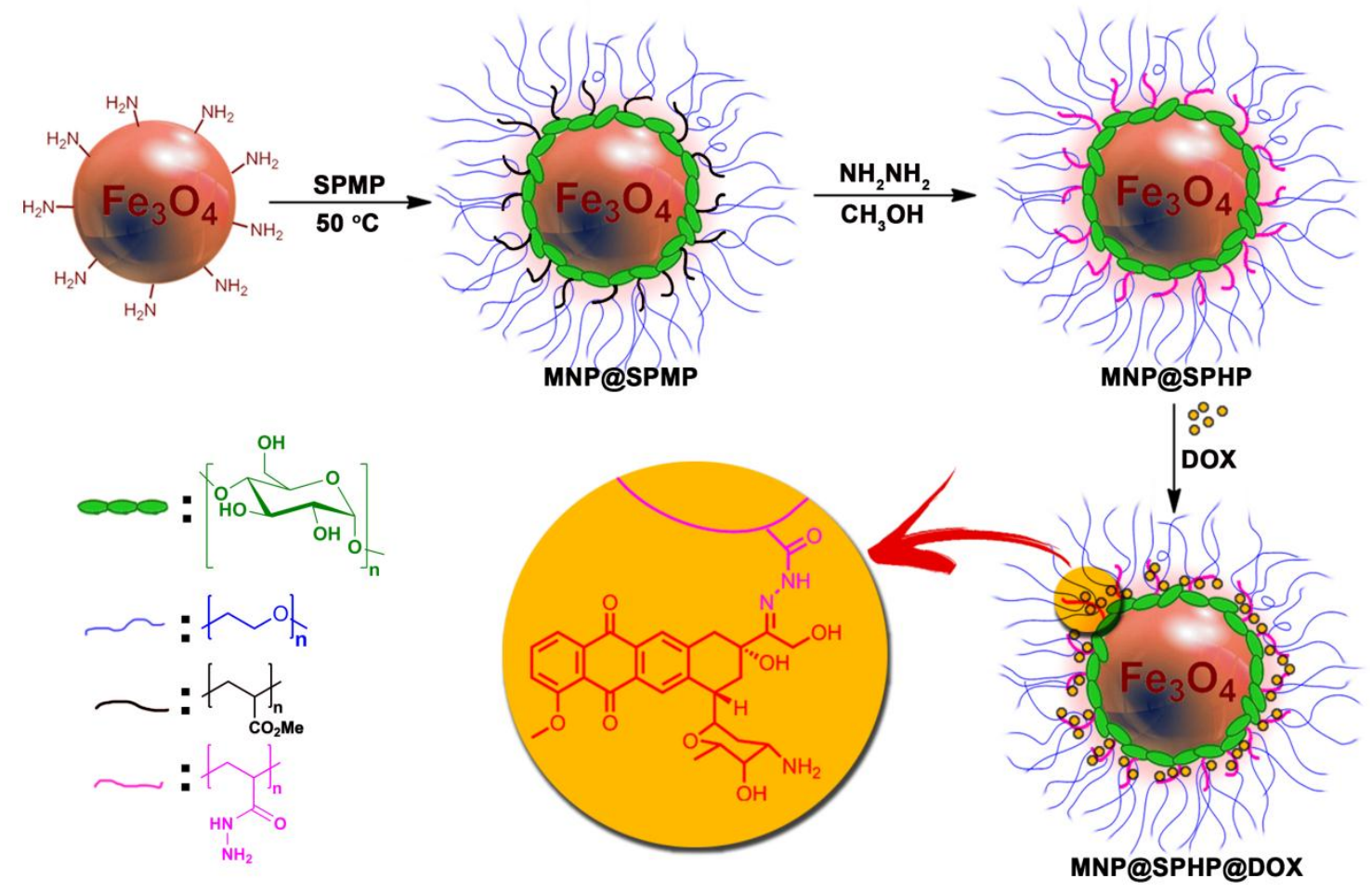

Scheme 2 Preparation steps of drug loaded magnetic nanocarrier MNP@SPHP@DOX

The GPC curves of starch and SPMP is shown in Figure S1. Compared to pure starch, the peak of SPMP shifted to higher molecular weight which confirms that copolymer was successfully grafted to starch. Also, only a single peak is observed in TGA curves of SPMP which shows that 
synthesized SPMP is almost pure and no homopolymer and non-grafted starch are presented in the sample. The results of GPC analysis showed that $M_{\mathrm{n}}$ of starch and SPMP are 34500 and $48200 \mathrm{~g} \mathrm{~mol}^{-1}$, respectively.

To confirm the structure of SPMP polymer, further investigation was performed using FT-IR spectrometer. The FT-IR spectra of starch, PEG-Am monomer and SPMP polymer were presented in Figure S2. The pure starch shows characteristic peaks at 982, 1465, 1646 and 2927 $\mathrm{cm}^{-1}$ which are attributed to stretching and bending vibration bands of $\mathrm{C}-\mathrm{O}, \mathrm{C}-\mathrm{C}$ and $\mathrm{C}-\mathrm{H}$. The FT-IR spectrum of macromonomer PEG-Am shows four main peaks at 1112, 1467, 1689 and $2885 \mathrm{~cm}^{-1}$ which is corresponded to stretching vibration of $\mathrm{C}-\mathrm{O}, \mathrm{C}=\mathrm{C}, \mathrm{C}=\mathrm{O}$ and $\mathrm{C}-\mathrm{H}$. Comparing to the FT-IR spectra of starch and PEG-Am with SPMP polymer, all characteristic peaks of starch and PEG-Am can be seen in FT-IR spectra of SPMP. Moreover, a strong peak at $1740 \mathrm{~cm}^{-}$ ${ }^{1}$ is attributed to $\mathrm{CO}_{2} \mathrm{Me}$ groups of poly(methyl methacrylate) in grafted copolymer. These results show that SPMP is successfully synthesized.

The FT-IR spectra were employed to demonstrate the successful coating of MNPs by SPMP polymer. Figure 1 shows the FT-IR spectra of $\mathrm{MNP} @ \mathrm{NH}_{2}$, SPMP, MNP@SPMP and MNP@SPHP. The FT-IR spectrum of MNP@ $\mathrm{NH}_{2}$ show vibration bands of Fe-O, Si-O, C-N, O$\mathrm{H}$ and $\mathrm{C}-\mathrm{H}$ at 589, 1016, 1384, 1625 and 2926, respectively. After coating of SPMP polymer onto the MNP surface same peaks as MNP@ $\mathrm{NH}_{2}$ and SPMP are observed in the FT-IR spectrum of MNP@SPMP. This result shows that SPMP is coated onto the MNPs surface. After reaction of MNP@SPMP with hydrazine the characteristic peak of methyl ester group is disappeared, which means that ester groups are successfully converted to hydrazon groups. In the FT-IR spectrum of MNP@SPHP, peaks at wavenumbers 589, 1022, 1631 and 2919 are attributed to Fe$\mathrm{O}, \mathrm{C}-\mathrm{O}, \mathrm{C}=\mathrm{O}$ and $\mathrm{C}-\mathrm{H}$, respectively. 


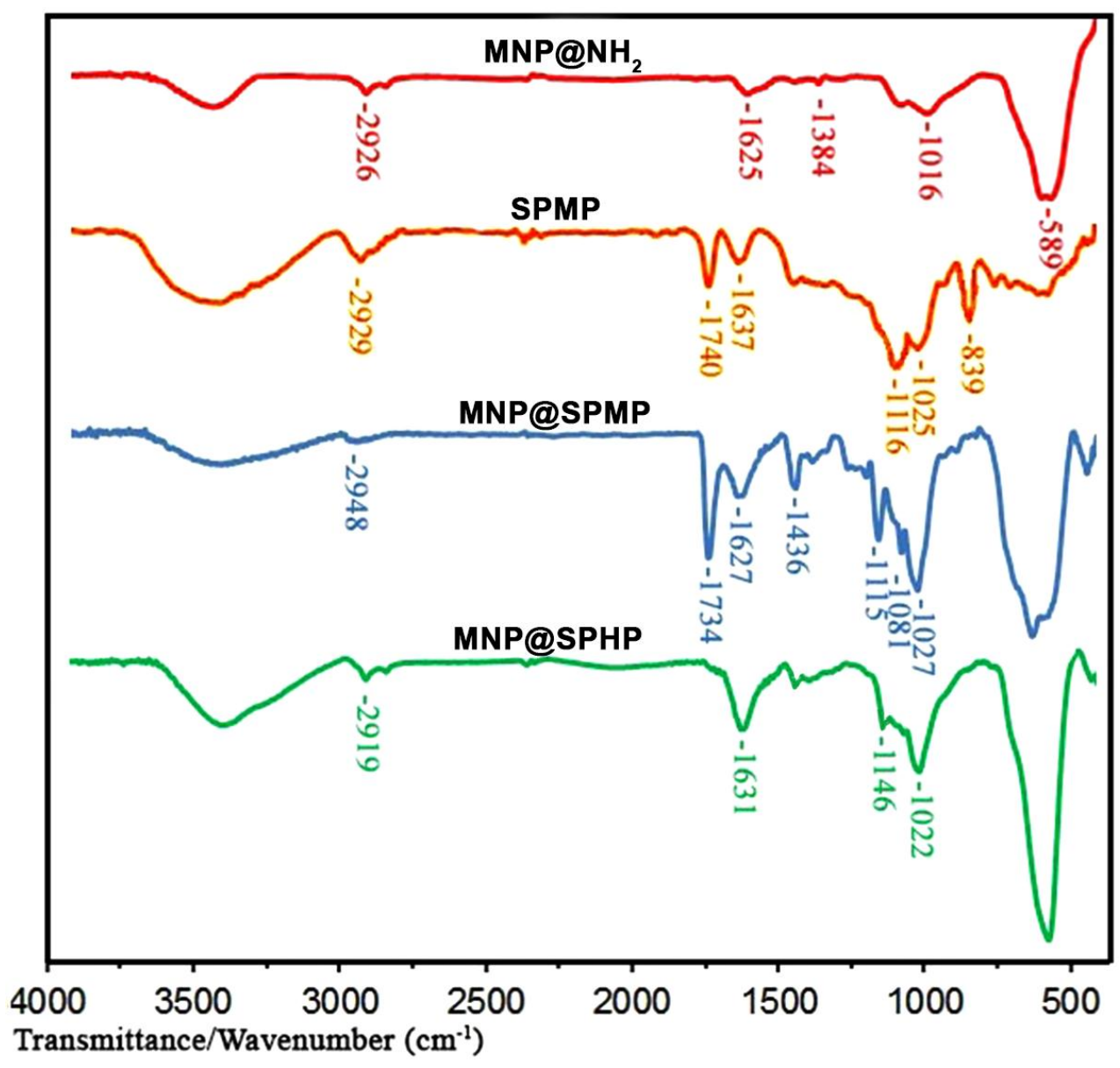

FIGURE 1 The FT-IR spectra of MNP@ $\mathrm{NH}_{2}$, SPMP polymer, MNP@SPMP and MNP@SPHP.

The TGA analysis was performed to confirm the grafting of copolymer to starch. As seen in Figure 2 TGA curve of starch shows a main weight loss at $220{ }^{\circ} \mathrm{C}$. The TGA curve of SPMP polymer shows different pattern and this polymer degraded in more than one step which is attributed to degradations of starch, methyl methacrylate and PEG groups. The TGA analysis also shows the amount of SPMP polymer onto the surface of MNP@SPMP. Based on the TGA curve of MNP@SPMP amount of coated SPMP polymer onto the surface of MNP is about $15 \%$. Moreover, the TGA curve of MNP@SPMP show same degradation pattern as SPMP. The degradation started at $230{ }^{\circ} \mathrm{C}$ and all of organic component degraded above $400{ }^{\circ} \mathrm{C}$. 


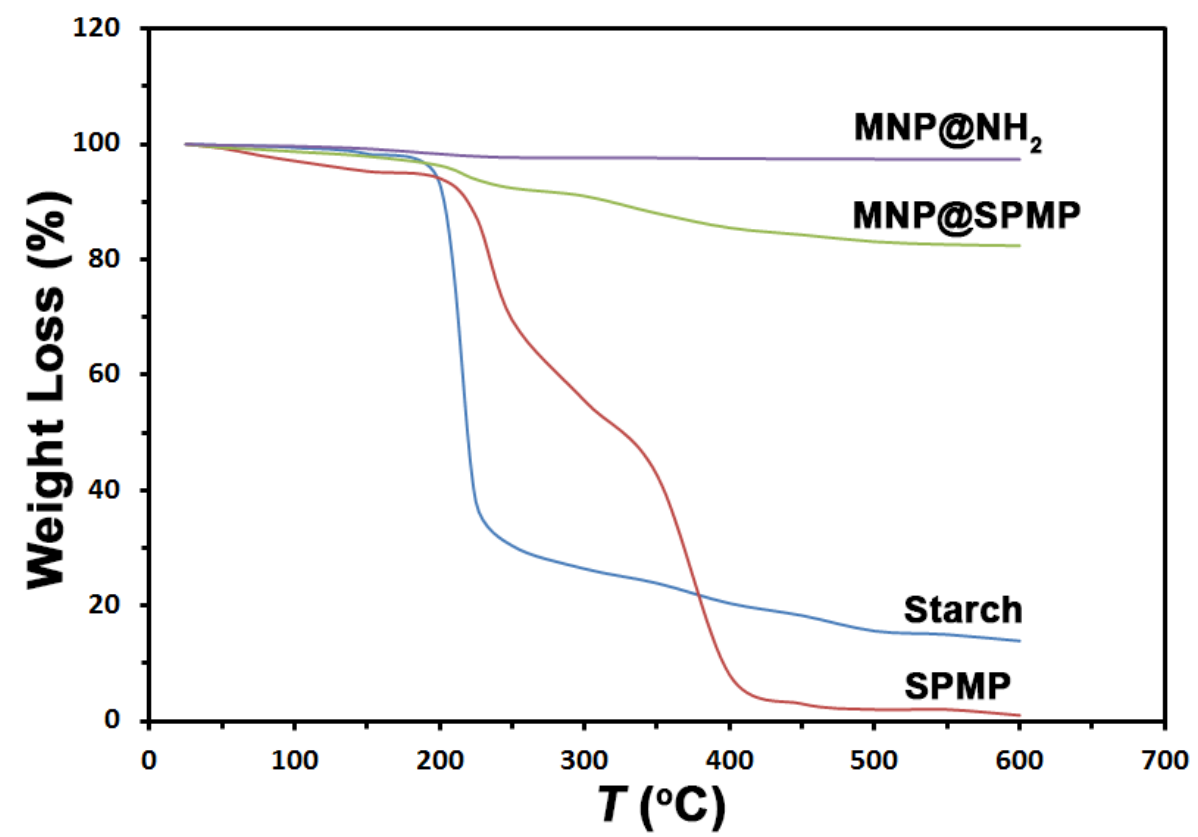

FIGURE 2 The TGA curves of MNP@ $\mathrm{NH}_{2}$, MNP@SPMP, Starch, SPMP polymer.

Figure S3 shows the XRD pattern of MNP@SPMP. It can be seen that MNP@SPMP shows the crystalline lines corresponded to cubic $\mathrm{Fe}_{3} \mathrm{O}_{4}$ (blue lines). The weak intensity of XRD pattern of MNP@SPMP is due to the coating of amorphous polymer onto the surface of MNPs. However, the peak positions are completely matched with $\mathrm{Fe}_{3} \mathrm{O}_{4}$ structure.

TEM image of MNP@SPHP shows almost spherical magnetic nanoparticles which are coated by polymer shells (Figure 3a). In the TEM image we can obviously see some aggregation of magnetic carrier which could be due to evaporation of solvent during the sample preparation for TEM analysis. From the TEM image the size of MNP@SPHP is about $19 \mathrm{~nm}$, while DLS analysis shows mean diameter of $93 \mathrm{~nm}$ (Figure 3b). It is worth noted that the observed DLS particle sizes are all larger than those measured by TEM. This is due to the fact that DLS measures the solvated nanoparticles in aqueous solution. These results are in agreement with previously published paper. ${ }^{51}$ 

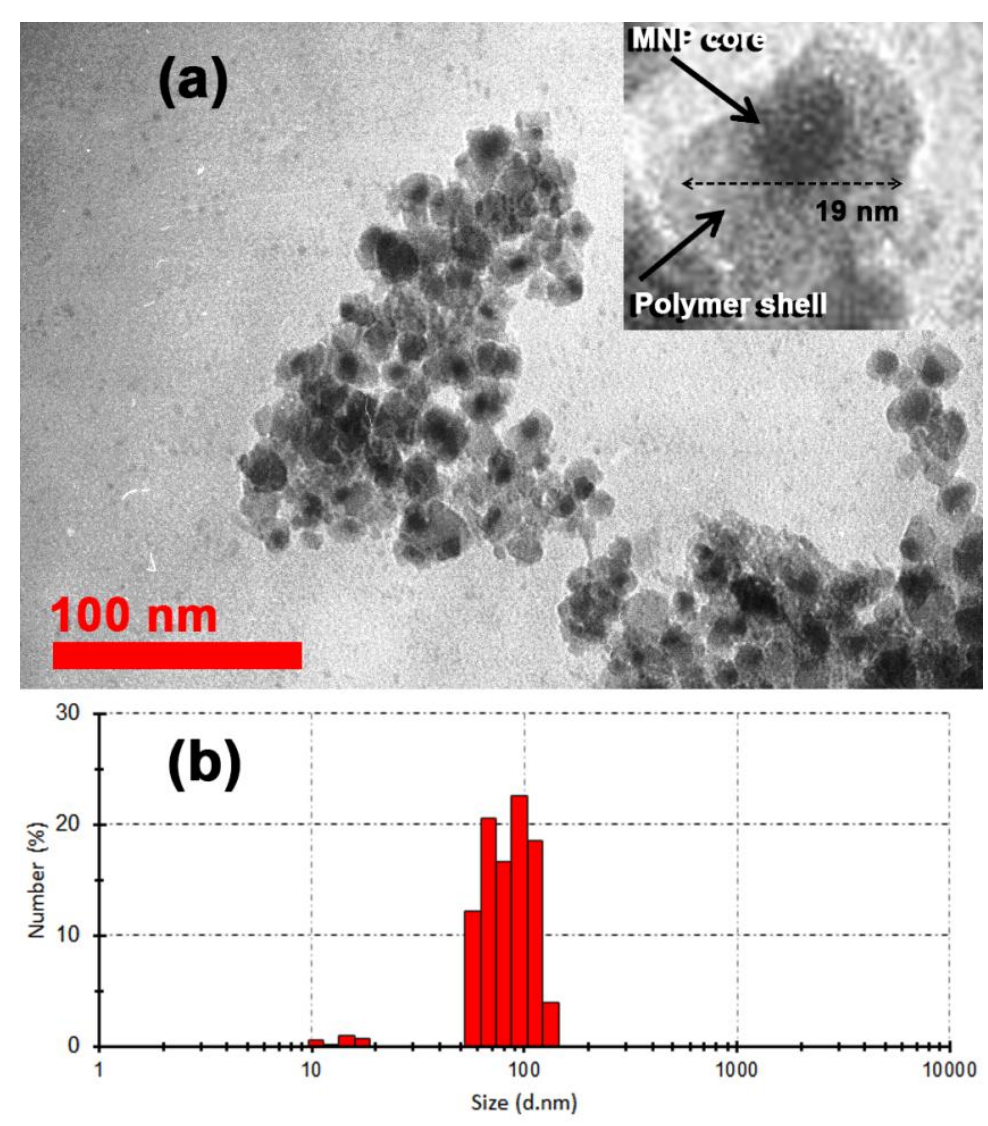

FIGURE 3 TEM (a) and DLS (b) image of MNP@SPHP

The surface charges of nanoparticles are a helpful parameter that can be used to characterize dispersion of colloidal in biological medium. Since, cell membranes are charged; it also plays an especially key role in cell-nanoparticle interactions. Zeta potential analysis of bare MNP, MNP@ $\mathrm{NH}_{2}, \mathrm{MNP} @ S P M P, M N P @ S P H P$ and MNP@SPHP@DOX was measured at pH=7.4 and the results are showed in Figure S4. As seen, bare MNP shows a highly negative surface charge $(-33.12 \pm 2.07 \mathrm{mV})$ due to MNP was prepared in alkaline solution. After coating of MNP with amine groups, surface charge increased to $-0.01 \pm 0.15 \mathrm{mV}$. After polymer coating the surface charges of MNP@SPMP and MNP@SPHP are +5.88 and +12.13 mV, respectively. 
After loading of DOX onto the MNP@SPHP the charge of surface slightly increased to $+14.07 \pm 0.85$, confirming that DOX was successfully loaded onto the surface of magnetic carrier. The magnetization property of nanocarrier is necessary for their practical applications. The magnetic hysteresis curves of MNP and MNP@SPHP were measured at $37{ }^{\circ} \mathrm{C}$ (Figure 4) and demonstrated that they are without remnant magnetization or coercivity, indicating that both samples are superparamagnetic at room temperature, which is characteristic of $\mathrm{Fe}_{3} \mathrm{O}_{4}$ nanoparticles. It also can be seen that magnetic carrier is dispersible and show good response to external magnet (Figure 4, inner image).

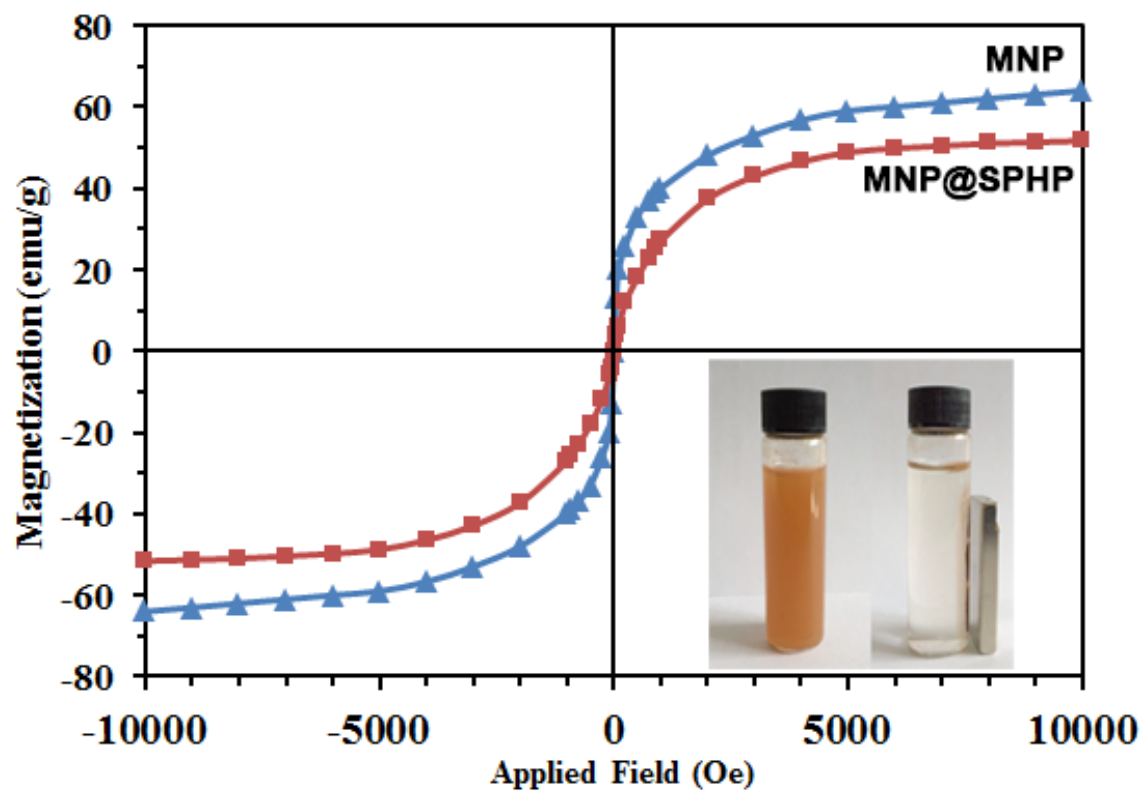

FIGURE 4 VSM analyses of MNP and MNP@SPHP

UV-vis spectroscopy was used to clarify the loading of DOX onto the MNP@SPHP nanocarrier (Figure 5a). Comparing the UV-vis spectra of bare MNP and MNP@SPHP showed that after coating of polymer onto the MNPs a peak at $400 \mathrm{~nm}$ is observed attributed to $\mathrm{n} \rightarrow \pi^{*}$ transition of carbonyl groups of SPHP. As seen, the absorption peak of free DOX is observed at $490 \mathrm{~nm}$. To 
reach the highest loading of drug on nanocarrier, $1 \mathrm{mg}$ of MNP@SPHP was mixed with $5 \mathrm{~mL}$ of various concentration of DOX solution $(30,50,70,90,110 \mu \mathrm{g} / \mathrm{mL})$. The UV-Vis spectra of samples with different DOX content shows that a peak at $490 \mathrm{~nm}$ increases along with increasing the DOX amount confirming the adsorption of DOX onto the nanocarrier surface. The results showed that increasing in the concentration of DOX more than $90 \mu \mathrm{g} / \mathrm{mL}$ did not changed $\lambda_{490}$ in the spectrum of MNP@SPHP@DOX and maximum loading of DOX is obtained in this concentration. In addition, in this sample drug loading capacity and efficiency of DOX on MNP@SPHP@DOX was determined 327士9.5 $\mathrm{mg} \mathrm{g}^{-1}$ and 72.6\%, respectively (Figure 5b). The high loading capacity of DOX on MNP@SPHP resulted from numerous hydrazine linkages on the surface of nanocarrier which easily grabs DOX from the solution.
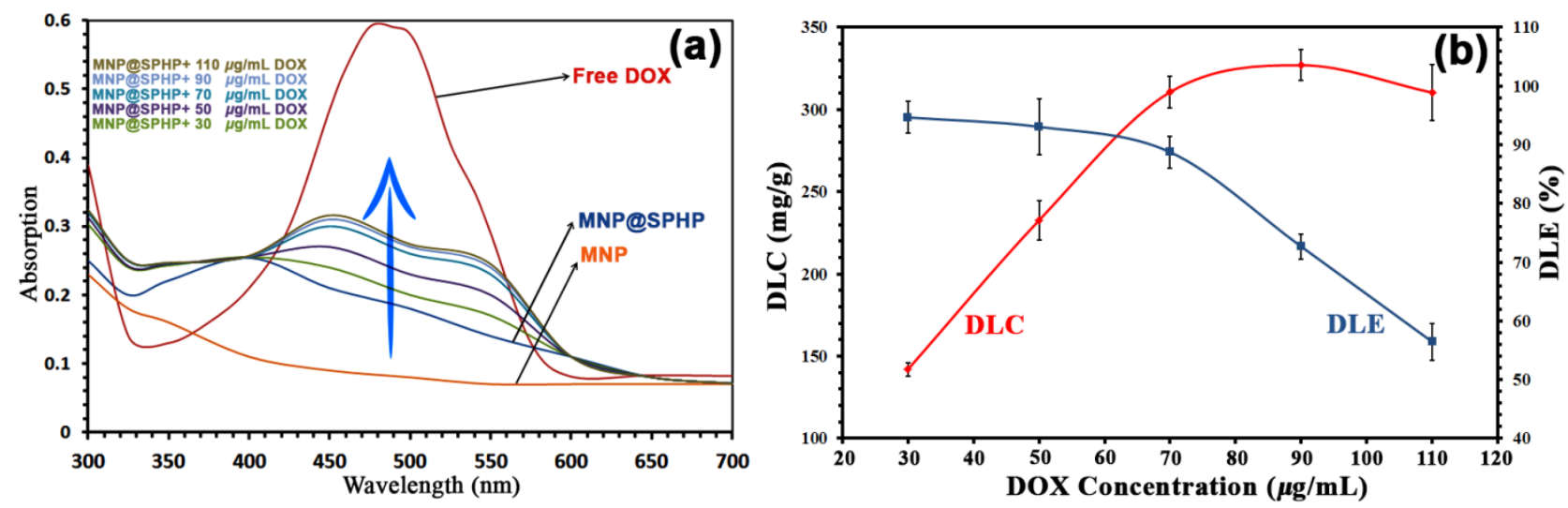

FIGURE 5 (a) UV-Vis spectra of MNP, MNP@SPHP, MNP@SPHP@DOX with various concentration of loaded DOX and free DOX, (b) DLC and ELC curves vs. DOX concentration

The in vitro release of DOX from MNP@SPHP@DOX was investigated at pH 5.0 (acetate buffer) and 7.4 (PBS buffer) at $37{ }^{\circ} \mathrm{C}$ (Figure 6). The DOX release profile shows that in neutral medium (PBS buffer) only $11 \%$ of DOX is detached from the carrier surface and released to solution. However, in acidic media (acetate buffer) more than $78 \%$ of loaded DOX is released to 
solution. Moreover, it can be seen that more than 50\% of conjugated DOX is released from nanocarrier over the first $24 \mathrm{~h}$ which shows fast responsiveness of nanocarrier to $\mathrm{pH}$ of environment. These results demonstrate that the hydrazone linkage in MNP@SPHP@DOX is stable under physiological condition $(\mathrm{pH}=7.4)$ while in acidic medium this linkage easily hydrolyzed and DOX detached from the surface of nanocarrier. Based on the results of drug release profile it can be deduced that drug release of MNP@SPHP@DOX is efficiently pHresponsive and DOX can be efficiently released in target sites from MNP@SPHP@DOX.

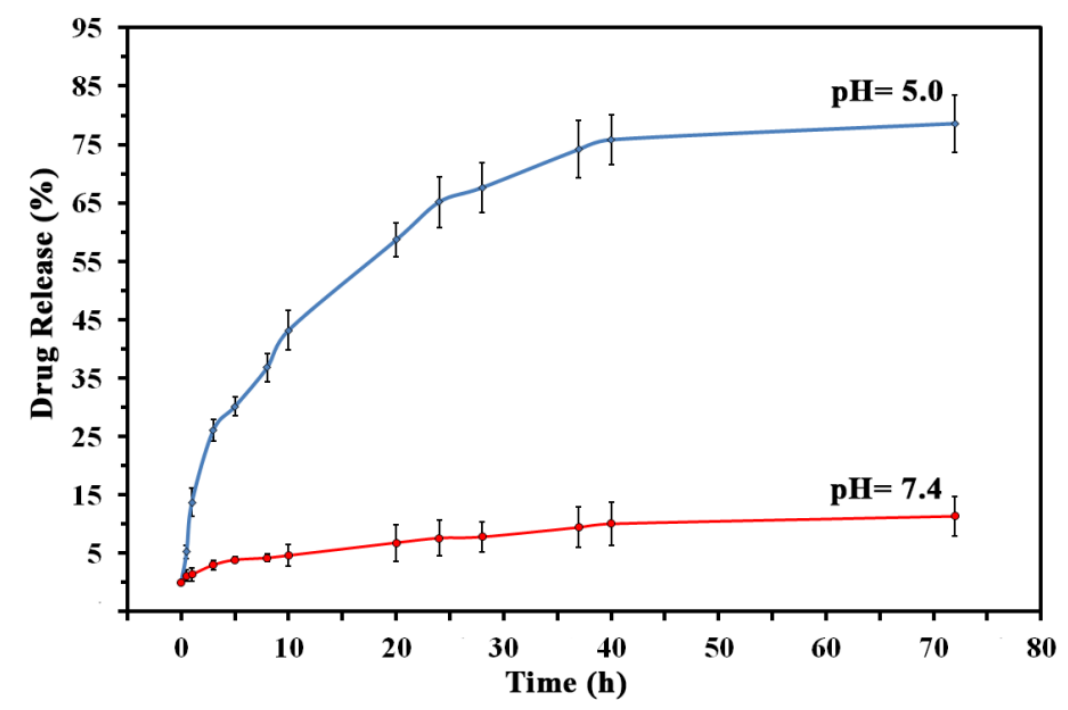

FIGURE 6 Cumulative release curves of DOX from the MNP@SPHP@DOX under different $\mathrm{pH}$ values at $37^{\circ} \mathrm{C}$.

The in vitro toxicity of the nanocarrier was checked towards HeLa cell lines using MTT assay (Figure 7). In this experiment free DOX was used as positive control. It was observed that the various concentrations of MNP@SPHP have no significant toxicity and showed satisfactory biocompatibility even at highest concentration. For MNP@SPHP@DOX, the viability of the cell is higher when the free DOX was added which can be attributed to lower concentrations of released DOX from MNP@SPHP@DOX, than concentrations of free DOX. Nevertheless, 
increasing in the concentration of MNP@SPHP@DOX to $50 \mu \mathrm{g} \mathrm{mL}^{-1}$ decreases the cell viability to less than 30\%. The higher toxicity of free DOX than MNP@SPHP@DOX can be explained by this fact that free DOX molecules are imported to cells by passive diffusion mechanism, while MNP@SPHP@DOXs should be firstly captured by cells trough a possible endocytosis mechanism and therefore DOX molecules are gradually released into cells. This is in agreement with previous reports. ${ }^{52-54}$ The results show that MNP@SPHP@DOX has excellent toxicity and can be used in vivo for cancer therapy.

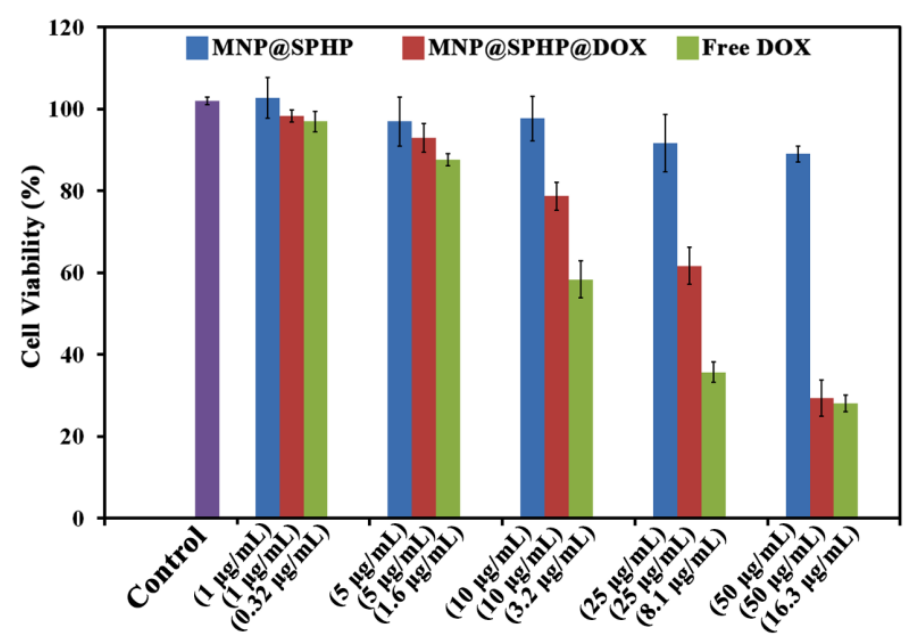

FIGURE 7 Cell viability of HeLa cells incubated with different concentrations of Free DOX, MNP@SPHP and MNP@SPHP@DOX for 24 h.

\section{CONCLUSION}

In summary, we have prepared a novel magnetic nanocarrier via facile and economical procedure. The magnetic nanocarrier was synthesized by coating of hydrazine modified starch onto the $\mathrm{Fe}_{3} \mathrm{O}_{4}$ magnetic nanoparticles. Moreover, coated polysaccharide was also attached to PEG molecules to increase the biocompatibility and biodistribution of nanocarrier in blood circulation. The resulted magnetic nanocarriers were then loaded by DOX as a model anti-cancer 
drug molecule. Due to the presence of numerous hydrazine linkages on the surface of nanocarrier, large amounts of DOX was covalently attached and loaded onto the magnetic carrier (327 mg g ${ }^{-1}$ ). The in vitro drug release profile of DOX loaded magnetic carrier shows that the presented carrier is strongly $\mathrm{pH}$-sensitive and only $11 \%$ of DOX is released under neutral condition (normal cell simulation) while more than 78\% DOX was released at acidic pH (cancer cell simulation). Also, MTT assay demonstrates that the DOX loaded magnetic carrier has effective cytotoxicity toward HeLa cells, while nanocarrier without DOX has no such toxicity even at high concentrations. The results show that the presented magnetic nanocarrier has good potential for targeted cancer therapy and it also can be used for MRI imaging.

\section{ACKNOWLEDGMENTS}

We are grateful for financial support from Iran National Science Foundation (INSF) and Iran's National Elites Foundation.

\section{REFERENCES}

1. C. Sun, J. S. Lee, M. Zhang. Adv. Drug Deliv. Rev. 2008, 60, 1252-1265.

2. O. Veiseh, J. W. Gunn, M. Zhang. Adv. Drug Deliv. Rev. 2010, 62, 284-304.

3. J. Dobson. Drug Dev. Res. 2006, 67, 55-60.

4. M. K. Jaiswal, M. Gogoi, H. D. Sarma, R. Banerjee, D. Bahadur. Biomater. Sci. 2014, 2, 370380.

5. C. S. Kumar, F. Mohammad. Adv. Drug Deliv. Rev. 2011, 63, 789-808. 
6. B. Chertok, B. A. Moffat, A. E. David, F. Yu, C. Bergemann, B. D. Ross, V. C. Yang. Biomaterials 2008, 29, 487-496.

7. V. V. Mody, A. Cox, S. Shah, A. Singh, W. Bevins, H. Parihar. Appl. Nanosci. 2014, 4, 385392.

8. L. H. Reddy, J. L. Arias, J. Nicolas, P. Couvreur. Chem. Rev. 2012, 112, 5818-5878.

9. K. Cheng, Z. Sun, Y. Zhou, H. Zhong, X. Kong, P. Xia, Z. Guo, Q. Chen. Biomater. Sci. 2013, $1,965-974$.

10. L. Gonzalez-Moragas, S.-M. Yu, E. Carenza, A. Laromaine, A. Roig. ACS Biomater. Sci. Eng. 2015, 1, 1129-1138.

11. H. J. Chung, H. Lee, K. H. Bae, Y. Lee, J. Park, S.-W. Cho, J. Y. Hwang, H. Park, R. Langer, D. Anderson. ACS nano 2011, 5, 4329-4336.

12. J. H. Lee, Y. w. Jun, S. I. Yeon, J. S. Shin, J. Cheon. Angew. Chem. 2006, 118, 8340-8342.

13. A. Singh, S. K. Sahoo. Drug Discov. Today 2014, 19, 474-481.

14. Z. Cheng, Y. Dai, X. Kang, C. Li, S. Huang, H. Lian, Z. Hou, P. Ma, J. Lin. Biomaterials 2014, 35, 6359-6368.

15. F. Ye, Å. Barrefelt, H. Asem, M. Abedi-Valugerdi, I. El-Serafi, M. Saghafian, K. Abu-Salah, S. Alrokayan, M. Muhammed, M. Hassan. Biomaterials 2014, 35, 3885-3894.

16. H. Wang, J. Shen, Y. Li, Z. Wei, G. Cao, Z. Gai, K. Hong, P. Banerjee, S. Zhou. Biomater. Sci. 2014, 2, 915-923.

17. N. Kohler, C. Sun, A. Fichtenholtz, J. Gunn, C. Fang, M. Zhang. Small 2006, 2, 785-792.

18. M. Arruebo, R. Fernández-Pacheco, M. R. Ibarra, J. Santamaría. Nano today 2007, 2, 22-32.

19. M. Torkpur-Biglarianzadeh, M. Salami-Kalajahi. RSC Adv. 2015, 5, 29653-29662.

20. A. Pourjavadi, Z. M. Tehrani, S. H. Hosseini. RSC Adv. 2015, 5, 48586-48595.

21. A. Pourjavadi, S. H. Hosseini, M. Alizadeh, C. Bennett. Colloid. Surface. B: Biointerf. 2014, $116,49-54$.

22. I. Urries, C. Muñoz, L. Gomez, C. Marquina, V. Sebastian, M. Arruebo, J. Santamaria. Nanoscale 2014, 6, 9230-9240.

23. D. Ling, N. Lee, T. Hyeon. Acc. Chem. Res. 2015.

24. Y. Li, R. Lin, L. Wang, J. Huang, H. Wu, G. Cheng, Z. Zhou, T. MacDonald, L. Yang, H. Mao. J. Mater. Chem. B 2015, 3, 3591-3603.

25. M. Hałupka-Bryl, M. Bednarowicz, B. Dobosz, R. Krzyminiewski, T. Zalewski, B. Wereszczyńska, G. Nowaczyk, M. Jarek, Y. Nagasaki. J. Magn. Magn. Mater. 2015, 384, 320327.

26. K. Kaaki, K. Hervé-Aubert, M. Chiper, A. Shkilnyy, M. Soucé, R. Benoit, A. Paillard, P. Dubois, M.-L. Saboungi, I. Chourpa. Langmuir 2011, 28, 1496-1505.

27. M. H. El-Dakdouki, D. C. Zhu, K. El-Boubbou, M. Kamat, J. Chen, W. Li, X. Huang. Biomacromolecules 2012, 13, 1144-1151.

28. A. Pourjavadi, Z. M. Tehrani, C. Bennett. Int. J. Polym. Mater. Polym. Biomater. 2015, 64, 570-577.

29. M. Guo, Y. Yan, H. Zhang, H. Yan, Y. Cao, K. Liu, S. Wan, J. Huang, W. Yue. J. Mater. Chem. 2008, 18, 5104-5112.

30. M. Calderón, M. A. Quadir, M. Strumia, R. Haag. Biochimie 2010, 92, 1242-1251.

31. V. Torchilin. Eur. J. Pharm. Biopharm. 2009, 71, 431-444.

32. R. Cheng, F. Meng, C. Deng, H.-A. Klok, Z. Zhong. Biomaterials 2013, 34, 3647-3657.

33. E. Fleige, M. A. Quadir, R. Haag. Adv. Drug Deliv. Rev. 2012, 64, 866-884.

34. S. Ganta, H. Devalapally, A. Shahiwala, M. Amiji. J. Control. Release 2008, 126, 187-204. 
35. S. Mura, J. Nicolas, P. Couvreur. Nature materials 2013, 12, 991-1003.

36. M. D. Howard, M. Jay, T. D. Dziubla, X. Lu. J. Biomed. Nanotech. 2008, 4, 133-148.

37. K. R. Chaudhari, M. Ukawala, A. S. Manjappa, A. Kumar, P. K. Mundada, A. K. Mishra, R. Mathur, J. Mönkkönen, R. S. R. Murthy. Pharm. Res. 2012, 29, 53-68.

38. A. J. Cole, A. E. David, J. Wang, C. J. Galbán, V. C. Yang. Biomaterials 2011, 32, 62916301.

39. E. M. Hoke, C. A. Maylock, E. Shacter. Free Radical Biol. Med. 2005, 39, 403-411.

40. X. Qi, W. Wei, J. Li, Y. Liu, X. Hu, J. Zhang, L. Bi, W. Dong. ACS Biomater. Sci. Eng. 2015.

41. A. Bast, H. Kaiserová, G. Den Hartog, G. Haenen, W. Van Der Vijgh. Cell Biol. Toxicol. 2007, 23, 39-47.

42. T. Petit. Bull. Cancer 2004, 91, 159-165.

43. V. Singh, P. Kumari, S. Pandey, T. Narayan. Bioresour. Technol. 2009, 100, 1977-1982.

44. A. Pourjavadi, S. H. Hosseini, S. T. Hosseini, S. A. Aghayeemeibody. Catal. Commun. 2012, $28,86-89$.

45. A. E.-H. Ali, A. AlArifi. Carbohydr. Polym. 2009, 78, 725-730.

46. S. Geresh, G. Y. Gdalevsky, I. Gilboa, J. Voorspoels, J. P. Remon, J. Kost. J. Control. Release 2004, 94, 391-399.

47. A. Rodrigues, M. Emeje. Carbohydr. Polym. 2012, 87, 987-994.

48. I. Y. Qudsieh, A. Fakhru'1-Razi, S. A. Muyibi, M. B. Ahmad, M. Ab Rahman, Z. W. Yunus, W. Md. J. Appl. Polym. Sci. 2004, 94, 1891-1897.

49. I. Mellman, R. Fuchs, A. Helenius. Annu. Rev. Biochem 1986, 55, 663-700.

50. F. Wang, Y.-C. Wang, S. Dou, M.-H. Xiong, T.-M. Sun, J. Wang. Acs Nano 2011, 5, 36793692.

51. H. Cai, X. An, J. Cui, J. Li, S. Wen, K. Li, M. Shen, L. Zheng, G. Zhang, X. Shi. ACS Appl. Mater. Interfaces 2013, 5, 1722-1731.

52. E. R. Gillies, J. M. Fréchet. Bioconjugate Chem. 2005, 16, 361-368.

53. S. Gai, P. Yang, D. Wang, C. Li, X. Li, N. Niu, J. Lin. J. Mater. Chem. 2011, 21, 1642016426.

54. Y. Hu, Y. Ding, D. Ding, M. Sun, L. Zhang, X. Jiang, C. Yang. Biomacromolecules 2007, 8, 1069-1076. 


\section{Graphical Abstract}

Hydrazine-Modified Starch Coated Magnetic Nanoparticles as an Effective pH-Responsive Nanocarrier for Doxorubicin Delivery $\uparrow$

Nasrin Zohreh, ${ }^{* a}$ Seyed Hassan Hosseini, ${ }^{\mathrm{b}}$ and Ali Pourjavadi ${ }^{\mathrm{b}}$

\section{Multifunctional Magnetic Nanocarrier \\ Large Amounts of Anti-Cancer Drug Loading \\ Highly pH-Sensetive Release of Drug}
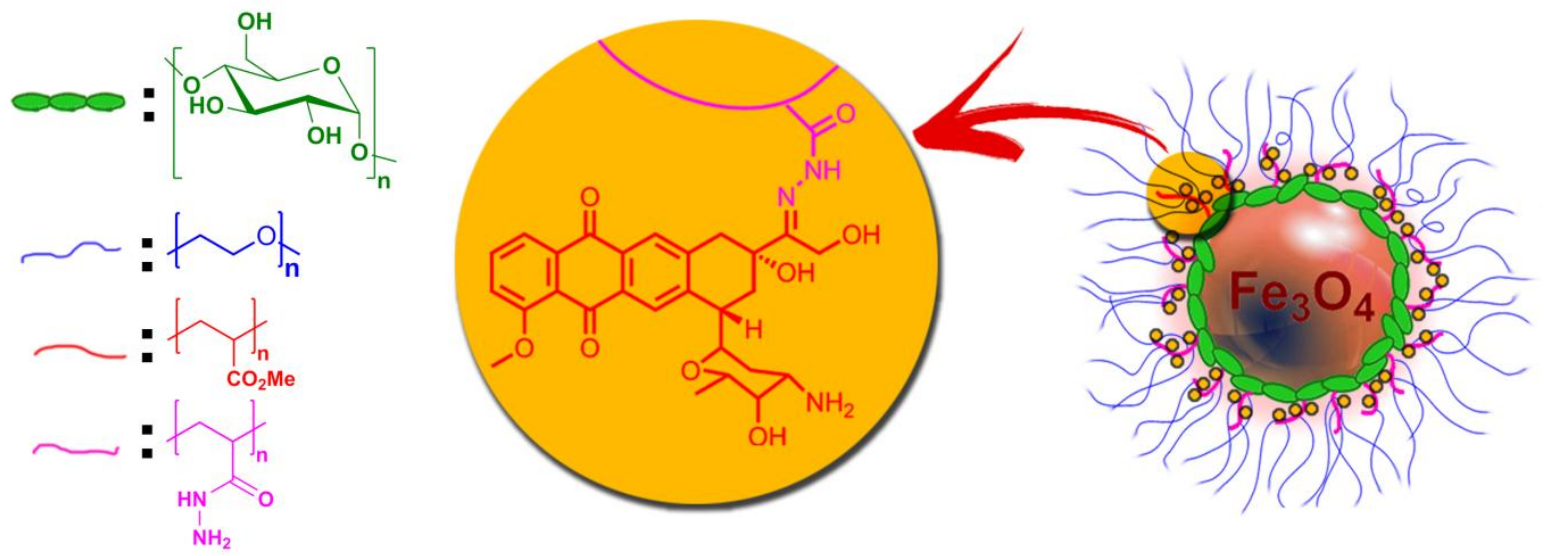